\title{
Morte Súbita na Doença de Parkinson: Qual o envolvimento do coração?
}

\author{
Sudden Unexpected Death in Parkinson's Disease: What is \\ the involvement of the heart?
}

\section{Muerte súbita en la enfermedad de Parkinson: ¿Cuál es la participación del corazón?}

\author{
Luis F. Sierra ${ }^{1}$, Noemi S. Araújo², Fúlvio A. Scorza ${ }^{3}$
}

\begin{abstract}
1.Zootecnista, Mestre em Ciências, Departamento de Neurologia e Neurocirurgia, Escola Paulista de Medicina, Universidade Federal de São Paulo, São Paulo-SP, Brasil.

2.Engenheira Biomédica, Mestre em Ciências, Departamento de Neurologia e Neurocirurgia, Escola Paulista de Medicina, Universidade Federal de São Paulo, São Paulo-SP, Brasil.

3.Docente, Doutor em Ciências, Departamento de Neurologia e Neurocirurgia, Escola Paulista de Medicina, Universidade Federal de São Paulo, São Paulo-SP, Brasil.
\end{abstract}

\begin{abstract}
Resumo
Introdução. A Doença de Parkinson (DP) é a segunda doença neurodegenerativa mais comum, trata-se de uma desordem neurológica progressiva, podendo atingir mais de $1 \% \mathrm{da}$ população acima de 60 anos, na maioria homens. A taxa de mortalidade de pacientes com DP é maior quando comparada com a população em geral. As causas dessa mortalidade elevada são desconhecidas, mas os fatores de risco cardiovascular podem desempenhar um papel importante. Nesse contexto, a morte súbita na doença de Parkinson (SUDPAR), corresponde a um evento importante que, embora raro, também ocorre. Objetivo. O objetivo dessa revisão é apresentar os principais achados na literatura quanto à relação entre anormalidades cardiovasculares e disfunções autonômicas com a morte súbita na DP. Método. Realizou-se pesquisas nas bases de dados Scielo, Google scholar e PubMed pelo termo Parkinson's disease em combinação com os termos (i) sudden death, (ii) sudden unexpected death, (iii) SUDPAR, (iv) cardiac abnormalities e ( $v$ ) autonomic disfunctions. Todas as publicações relevantes foram consideradas. Resultados. Existem ainda poucos estudos na literatura sobre SUDPAR, sendo que, de 2006 a 2020, foram publicados 22 trabalhos entre cartas ao editor, artigos originais, revisões e relatos de caso. Pesquisas clínicas e experimentais sugerem que anormalidades cardíacas e disfunções autonốmicas desempenham um papel fundamental. Conclusão. Diversos estudos apresentam o envolvimento cardíaco na DP, sendo que disfuncõoes autonômicas são causas frequentes de morte em pacientes com essa doença. A colaboração entre médicos e pesquisadores é fundamental para identificar abordagens que, considerando a investigação cardiológica, ofereçam a possibilidade de prevenção da SUDPAR.
\end{abstract}

Unitermos. Doença de Parkinson; morte súbita; SUDPAR; anormalidades cardíacas; disfunç̃ões autonômicas

\begin{abstract}
Introduction. Parkinson's disease (PD) is the second most common neurodegenerative disease. It is a progressive neurological disorder, reaching more than $1 \%$ of the population over 60 years, mostly men. The mortality rate of patients with PD is higher when compared to the general population. The causes of this high mortality are unknown, but cardiovascular risk factors can play an important role. Thus, sudden unexpected death in Parkinson's disease (SUDPAR), corresponds to an important event that, although rare, also occurs. Objective. The purpose of this review is to present the main findings in the literature regarding the relationship between cardiovascular and autonomic dysfunctions with sudden death in PD. Method. The research was carried out in the Scielo, Google scholar and PubMed databases for the term Parkinson's disease in combination with the terms (i) sudden death, (ii) sudden unexpected death, (iii) SUDPAR, (iv) cardiac abnormalities and ( $v$ ) autonomic disfunctions. All relevant publications were considered. Results. There are still few studies in the literature on
\end{abstract}


SUDPAR, and from 2006 to 2020, 22 papers were published, including letters to the editor, reviews and case reports. Clinical and experimental research suggests that cardiac abnormalities and autonomic dysfunctions play a key role. Conclusion. Several studies have shown cardiac involvement in PD, and autonomic dysfunctions are frequent causes of death in patients with this disease. The collaboration between doctors and researchers is essential to identify approaches that, considering cardiological research, offer the possibility of preventing SUDPAR.

Keywords. Parkinson's disease; sudden death; SUDPAR; cardiac abnormalities; autonomic dysfunctions

\begin{abstract}
Resumen
Introducción. La enfermedad de Parkinson (EP) es la segunda enfermedad neurodegenerativa más común, es un trastorno neurológico progresivo, llegando a más del $1 \%$ de la población mayor de 60 años, en su mayoría hombres. La tasa de mortalidad de los pacientes con EP es mayor en comparación con la población general. Se desconocen las causas de esta alta mortalidad, pero los factores de riesgo cardiovascular pueden desempeñar un papel importante. Así, la muerte súbita en la enfermedad de Parkinson (SUDPAR), corresponde a un evento importante que, aunque raro, también ocurre. Objetivo. El propósito de esta revisión es presentar los principales hallazgos de la literatura sobre la relación entre las disfunciones cardiovasculares y autonómicas con la muerte súbita en la EP. Método. La investigación se llevó a cabo en las bases de datos Scielo, Google Scholar y PubMed para el término enfermedad de Parkinson en combinación con los términos (i) muerte súbita, (ii) muerte súbita inesperada, (iii) SUDPAR, (iv) anomalías cardíacas y ( $v$ ) disfunciones autonómicas. Se consideraron todas las publicaciones relevantes. Resultados. Aún existen pocos estudios en la literatura sobre el tema, y de 2006 a 2020 se publicaron 22 artículos, entre cartas al editor, revisiones y reportes de casos. La investigación clínica y experimental sugiere que las anomalías cardíacas y las disfunciones autónomas desempeñan un papel clave. Conclusión. Varios estudios han demostrado la afectación cardíaca en la EP y las disfunciones autonómicas son causas frecuentes de muerte en pacientes con esta enfermedad. La colaboración entre médicos e investigadores es fundamental para identificar enfoques que, considerando la investigación cardiológica, ofrezcan la posibilidad de prevenir la SUDPAR.
\end{abstract}

Palabras clave. Enfermedad de Parkinson; muerte súbita; SUDPAR; anomalías cardíacas; disfunciones autonómicas

Trabalho desenvolvido para o curso "Neurociência em Pauta" ("Neuroscience at hand"), ministrado pelo Programa de Pós-Graduação em Neurologia/Neurociências da Escola Paulista de Medicina, Unifesp, São Paulo-SP, Brasil.

\title{
INTRODUÇÃO
}

A Doença de Parkinson (DP) foi descrita pela primeira vez por James Parkinson, em 1817, na Inglaterra. Trata-se da segunda doença neurodegenerativa mais comum, ficando atrás apenas da doença de Alzheimer ${ }^{1}$.

A DP é caracterizada por sintomas motores e não motores, afetando duas a cada mil pessoas em qualquer momento da vida, e segue aumentando sua prevalência com a idade, podendo atingir $1 \%$ da população com mais de 60 
anos, homens na sua maioria. As causas da DP ainda são desconhecidas na maioria dos pacientes, e podem incluir, dentre outros, fatores genéticos, os quais correspondem a 5 $10 \%$ dos $\operatorname{casos}^{2,3}$. Além disso, diversos estudos relacionam a DP com fatores ambientais e comportamentais, tais como consumo de produtos lácteos, exposição à pesticidas, histórico de melanomas, lesão cerebral traumática e consumo de tabaco e cafeína ${ }^{4,5}$. Diversos medicamentos auxiliam nas funções motoras do dia a dia realizadas por pacientes parkinsonianos, porém, para muitos desses pacientes os efeitos dessas drogas não são tão evidentes ou podem causar efeitos colaterais adversos ${ }^{6}$.

Mesmo com os recentes avanços na compreensão dessa doença, os fatores de risco relacionados à morte na DP ainda não foram completamente esclarecidos ${ }^{7}$. Estudos mostram que pacientes com DP apresentam maior risco de mortalidade prematura ${ }^{8-10}$, sendo que esse índice pode atingir um valor duas vezes maior em comparação à população geral ${ }^{11}$. Nesse contexto, embora pouco descrita, a morte súbita na doença de Parkinson (sudden unexpected death in Parkinson's disease, SUDPAR), corresponde a um evento importante que, mesmo raro, também ocorre ${ }^{7,12}$. Os mecanismos envolvidos na SUDPAR permanecem desconhecidos, contudo, estudos recentes apontam para anormalidades cardiovasculares e disfunções autonômicas cardíacas como fatores de risco relevantes ${ }^{12-16}$. O objetivo deste estudo é apresentar, por meio de revisão da literatura, os principais achados quanto à relaçãao entre disfunções 
cardiovasculares e autonômicas com a morte súbita na doença de Parkinson.

\section{MÉTODO}

Essa revisão foi desenvolvida através de pesquisas nas bases de dados Scielo, Google scholar e PubMed (MEDLINE). Utilizou-se a palavra-chave Parkinson's disease em combinação individual com os termos (i) sudden death, (ii) sudden unexpected death, (iii) SUDPAR, (iv) cardiac abnormalities e $(v)$ autonomic disfunctions. Considerou-se todos os estudos relevantes, incluindo artigos originais (clínicos e experimentais), relatos de caso, revisões, e cartas /comentário ao editor. Apenas artigos em inglês ou português foram avaliados.

\section{RESULTADOS E DISCUSSÃO}

A morte súbita na doença de Parkinson (SUDPAR) é definida como a morte inesperada de um paciente com DP, sem qualquer causa de morte evidente identificada na autópsia7,12. A investigação da SUDPAR é particularmente difícil, uma vez que esse é um evento raro e os exames postmortem, na maioria dos casos, são insuficientes ${ }^{7}$. Uma das primeiras descriç̃os de morte sem causa definida em pacientes com Parkinsonismo foi publicada em 1976 por Rajput e Rozdilsky ${ }^{17}$. Nesse relato, aproximadamente $16 \%$ dos pacientes morreram subitamente sem causa toxicológica ou anatômica identificável em autópsia ${ }^{17}$. Existem ainda poucos estudos na literatura sobre SUDPAR sendo que, de 
2006 a 2020, foram publicados 22 trabalhos entre cartas ao editor, artigos originais, revisões e relatos de caso (Tabela 1).

Embora os mecanismos subjacentes à morte súbita na DP permaneçam desconhecidos, pesquisas clínicas e experimentais sugerem que anormalidades cardíacas e disfunções autonômicas desempenham um papel fundamental ${ }^{13,14,16,18}$, uma vez que, aproximadamente $60 \%$ dos pacientes com DP apresentam distúrbios cardiovasculares $^{19}$. As disfunções autonômicas (ou disautonomia) também são frequentes nessa doença, sendo essa a anormalidade cardíaca de maior predominância ${ }^{19}$.

O envolvimento cardíaco na SUDPAR pode estar relacionado ao uso de medicamentos, tais como a domperidona. A domperidona consiste em um antagonista dopaminérgico preferencialmente utilizado no tratamento de sintomas gastrointestinais e hipotensão ortostática na DP, uma vez que o risco de efeitos adversos extrapiramidais é mínimo ${ }^{39-41}$. Contudo, seu uso pode aumentar o risco de taquiarritmia ventricular, morte súbita cardíaca (MSC) e causar o prolongamento do intervalo QT, particularmente em pacientes com histórico de doenças cardiovasculares ${ }^{40-43}$. Assim, o tratamento farmacológico na DP (notadamente, a polifarmácia) pode levar a anormalidades cardíacas as quais correlacionam-se positivamente com o desencadeamento da SUDPAR ${ }^{15,19}$. 
Tabela 1. Relação de estudos publicados nas bases de dados Scielo, Google Scholar e PubMed sobre morte súbita e doença de Parkinson.

\begin{tabular}{|c|c|c|}
\hline Autor/ano & $\begin{array}{c}\text { Tipo de } \\
\text { publicação }\end{array}$ & Principais achados/comentários \\
\hline $\begin{array}{l}\text { Sato et al., } \\
2006^{20}\end{array}$ & Original & $\begin{array}{l}5,3 \% \text { dos pacientes com DP avaliados morreram de morte } \\
\text { súbita }\end{array}$ \\
\hline $\begin{array}{l}\text { Matsumoto } \\
\text { et al., } \\
2014^{13} \\
\end{array}$ & Original & $\begin{array}{l}\text { Uma quantidade não desprezível de pacientes com DP morre } \\
\text { de morte súbita. Nesse estudo, } 25 \% \text { dos pacientes } \\
\text { acompanhados morreram de morte súbita }\end{array}$ \\
\hline $\begin{array}{l}\text { Wang et al., } \\
2015^{21}\end{array}$ & Original & $\begin{array}{l}\text { Identificação das taxas de mortalidade e preditores de morte } \\
\text { em pacientes com DP na China. Um entre } 11 \text { pacientes } \\
\text { morreu de morte súbita sem causa identificada }\end{array}$ \\
\hline $\begin{array}{l}\text { Scorza et } \\
\text { al., } 2016^{22}\end{array}$ & Carta & $\begin{array}{l}\text { Taxa de mortalidade e SUDPAR pode aumentar com a maior } \\
\text { incidência de DP }\end{array}$ \\
\hline $\begin{array}{l}\text { Scorza et } \\
\text { al., } 2017^{7}\end{array}$ & Revisão & $\begin{array}{l}\text { Apenas } 2 \% \text { dos estudos em DP nos últimos } 30 \text { anos referem- } \\
\text { se à mortalidade }\end{array}$ \\
\hline $\begin{array}{l}\text { Nishida et } \\
\text { al., } 2017^{23}\end{array}$ & $\begin{array}{l}\text { Relato de } \\
\text { caso }\end{array}$ & $\begin{array}{l}\text { Desregulação autonômica, alterações estruturais no coração e } \\
\text { arritmias podem aumentar o risco de SUDPAR }\end{array}$ \\
\hline $\begin{array}{l}\text { Scorza et } \\
\text { al., } 2017^{24}\end{array}$ & Carta & $\begin{array}{l}\text { Apneia Obstrutiva do Sono pode ser um fator de risco para } \\
\text { SUDPAR }\end{array}$ \\
\hline $\begin{array}{l}\text { Scorza et } \\
\text { al., } 2018^{25}\end{array}$ & Carta & SUDPAR surge como um problema clínico importante \\
\hline $\begin{array}{l}\text { Scorza et } \\
\text { al., } 2018^{26}\end{array}$ & Carta & $\begin{array}{l}\text { Rotina de avaliação cardiovascular em pacientes com DP pode } \\
\text { reduzir a frequência de SUDPAR }\end{array}$ \\
\hline $\begin{array}{l}\text { Scorza et } \\
\text { al., } 2018^{19}\end{array}$ & Revisão & $\begin{array}{l}\text { Disfunções autonômicas cardíacas são altamente incidentes } \\
\text { na DP e Parkinsonismo }\end{array}$ \\
\hline $\begin{array}{l}\text { Rodrigues et } \\
\text { al., } 2019^{27}\end{array}$ & $\begin{array}{l}\text { Artigo } \\
\text { original }\end{array}$ & $\begin{array}{l}\text { Modelo experimental de Parkinsonismo tratado com } \\
\text { domperidona apresentou comprometimento cardiovascular }\end{array}$ \\
\hline $\begin{array}{l}\text { Nejm et al., } \\
2019^{28}\end{array}$ & Carta & $\begin{array}{l}\text { Necessidade de definição apropriada de tópicos de pesquisa } \\
\text { em mortalidade na DP }\end{array}$ \\
\hline $\begin{array}{l}\text { Zhang et } \\
\text { al., } 2019^{29}\end{array}$ & Carta & $\begin{array}{l}\text { Regulação anormal do sistema nervoso autonômico devido à } \\
\text { patologia dos corpos de Lewy pode ser um fator de risco para } \\
\text { morte súbita em pacientes com DP }\end{array}$ \\
\hline $\begin{array}{l}\text { Nejm et al., } \\
2019^{30}\end{array}$ & Carta & $\begin{array}{l}\text { Importância da discussão entre neurologistas e pacientes } \\
\text { sobre a questão da SUDPAR }\end{array}$ \\
\hline $\begin{array}{l}\text { Menezes- } \\
\text { Rodrigues et } \\
\text { al., } 2019^{31} \\
\end{array}$ & Carta & Desidratação pode contribuir para a morte súbita na DP \\
\hline $\begin{array}{l}\text { Rodrigues et } \\
\text { al., } 2019^{32}\end{array}$ & Carta & $\begin{array}{l}\text { Aspectos prodrômicos da DP ganham destaque, } \\
\text { especialmente o transtorno comportamental do sono REM }\end{array}$ \\
\hline $\begin{array}{l}\text { Rodrigues et } \\
\text { al., } 2020^{33}\end{array}$ & Carta & $\begin{array}{l}\text { SUDPAR pode resultar de complicações na cardiomiopatia de } \\
\text { Takotsubo }\end{array}$ \\
\hline $\begin{array}{l}\text { Alves et al., } \\
2020^{34}\end{array}$ & Carta & $\begin{array}{l}\text { Não foi possível concluir que pacientes com DP e alto risco de } \\
\text { eventos coronários apresentarão maior risco de morte súbita }\end{array}$ \\
\hline $\begin{array}{l}\text { Scorza et } \\
\text { al., } 2020^{35}\end{array}$ & Carta & $\begin{array}{l}\text { O aumento de IL-6 está associado à doença cardíaca } \\
\text { coronária e a eventos fatais súbitos. Níveis elevados de IL-6 } \\
\text { podem indicar risco aumentado de mortalidade em pacientes } \\
\text { com DP }\end{array}$ \\
\hline $\begin{array}{l}\text { Rodrigues et } \\
\text { al., } 2020^{36}\end{array}$ & Carta & Possível relação causal entre hipotireoidismo e SUDPAR \\
\hline $\begin{array}{l}\text { Scorza et } \\
\text { al., } 2020^{37}\end{array}$ & Carta & $\begin{array}{l}\text { Sugestão para inclusão da hipertensão arterial dentre os } \\
\text { fatores de risco para SUDPAR }\end{array}$ \\
\hline Jost, $2020^{38}$ & Carta & $\begin{array}{l}\text { A frequência e a importância da hipertensão arterial em } \\
\text { pacientes com DP são subestimadas }\end{array}$ \\
\hline
\end{tabular}


Lertxundi et al (2013) alertaram sobre a cardiotoxicidade da domperidona na DP, especialmente em doses acima de $30 \mathrm{mg} / \mathrm{dia}^{40}$. Em um estudo retrospectivo multicêntrico, Renoux et al (2016) avaliaram a taxa de taquiarritmia ventricular e MSC associada ao uso de domperidona em pacientes com DP, e uma taxa de incidência de $1,35 \%$ (2907 casos entre 214.962 pacientes) foi identificada, além do risco aumentado da ocorrência de taquiarritmia ventricular e MSC nesses pacientes, independente da dose e/ou tempo de tratamento com domperidona ${ }^{16}$. Recentemente, os achados descritos por Rodrigues et al (2019) sugerem que a administração de domperidona em pacientes com DP pode desempenhar um papel na SUDPAR ${ }^{27}$. Nesse estudo, animais submetidos ao modelo de Parkinsonismo com 6-hidroxidopamina que receberam a dose máxima ajustada de domperidona em humanos apresentaram comprometimento cardiovascular significativo ${ }^{27}$. É preciso salientar que os pacientes com DP são majoritariamente idosos, podendo apresentar diversas comorbidades. As comorbidades associadas podem tornar os pacientes mais propensos a eventos adversos cardíacos relacionados ao uso da domperidona ${ }^{16,38}$.

Outra condição de envolvimento do coração na SUDPAR consiste na disfunção autonômica cardíaca. A disfunção autonômica é um fator de risco para o prolongamento do intervalo $\mathrm{QT}^{44-46}$, o qual, por sua vez, pode favorecer o desenvolvimento de torsades de pointes, taquicardia ventricular e morte súbita ${ }^{43}$. O intervalo QT reflete a duração 
total da despolarização e repolarização ventricular, sendo afetado pela complexa interação entre o sistema nervoso simpático e parassimpático ${ }^{40}$. Uma vez que o prolongamento do intervalo QT está associado a um limiar de fibrilação ventricular reduzido e arritmia ventricular, ele pode ser um fator preditivo para a morte súbita cardíaca na DP40,47. Em um estudo que avaliou a relação entre o prolongamento do intervalo QT corrigido por frequência (QTC) e a gravidade da disfunção autonômica na DP e doenças correlacionadas, os autores concluíram que as anormalidades no QTc podem refletir a degeneração dos neurônios simpáticos e parassimpáticos ${ }^{44}$. Curiosamente, o prolongamento do intervalo QT na DP parece não estar relacionado ao desequilíbrio eletrolítico, cardiopatia subjacente ou exposição à tratamento farmacológico propenso a aumentar esse intervalo ${ }^{43,44}$.

Sintomas cardiovasculares estão presentes em quase todos os estágios da DP ${ }^{19}$. Além das anormalidades cardíacas discutidas nessa revisão, os pacientes com DP podem desenvolver cardiomiopatia, doença cardíaca coronária, arritmias, hipertensão arterial e/ou apresentam um aumento da taxa de insuficiência cardíaca ${ }^{19,38}$. Contudo, embora uma quantidade significativa de trabalhos na literatura sustenta 0 envolvimento do coração na doença de Parkinson, essas evidências raramente são consideradas no manejo desses pacientes $^{19}$. O reconhecimento precoce do envolvimento cardíaco é fundamental para prevenir a morte súbita cardíaca e a SUDPAR. Os modelos experimentais de DP 
podem contribuir na investigação da fisiopatologia desse evento ${ }^{27}$.

\section{CONCLUSÃO}

Diversos estudos sugerem que a SUDPAR contribui de maneira importante para a mortalidade na DP. Embora os mecanismos fisiopatológicos subjacentes à SUDPAR ainda não foram elucidados, existe um corpo significativo da literatura que apresenta o envolvimento cardíaco na doença de Parkinson, sendo que sintomas cardíacos e cardiovasculares são causas frequentes de morte em pacientes com DP. Assim, médicos de várias especialidades e pesquisadores devem colaborar para estabelecer protocolos experimentais e clínicos para identificar abordagens que ofereçam a possibilidade de prevenção da morte súbita na doença de Parkinson, considerando a investigação cardiológica.

\section{REFERÊNCIAS}

1.Elbaz A, Carcaillon L, Kab S, Moisan F. Epidemiology of Parkinson's disease. Rev Neurologiq 2016;172:14-26. https://doi.org/10.1016/j.neurol.2015.09.012

2.Tysnes OB, Storstein A. Epidemiology of Parkinson's disease. J Neural Trans 2017;124:901-5. https://doi.org/10.1007/s00702-017-1686-y 3.Schneider RB, Iourinets J, Richard IH. Parkinson's disease psychosis: presentation, diagnosis and management. Neurodegen Dis Manag 2017;7:365-76. https://doi.org/10.2217/nmt-2017-0028

4.Kieburtz K, Wunderle KB. Parkinson's disease: Evidence for environmental risk factors. Mov Dis 2013;28:8-13. https://doi.org/10.1002/mds. 25150

5. Ascherio A, Schwarzschild MA. The epidemiology of Parkinson's disease: risk factors and prevention. Lancet Neurol 2016;15:1257-72. https://doi.org/10.1016/S1474-4422(16)30230-7 
6. Hayes MT. Parkinson's Disease and Parkinsonism. Am J Med 2019;132:802-7. https://doi.org/10.1016/j.amjmed.2019.03.001

7.Scorza FA, do Carmo AC, Fiorini AC, Nejm MB, Scorza CA, Finsterer $\mathrm{J}$, et al. Sudden unexpected death in Parkinson's disease (SUDPAR): a review of publications since the decade of the brain. Clinics 2017;72:649-51. https://doi.org/10.6061/clinics/2017(11)01

8. D'Amelio M, Ragonese P, Morgante L, Reggio A, Callari G, Salemi G, et al. Long-term survival of Parkinson's disease: A population-based study. J Neurol 2006;253:33-7. https://doi.org/10.1007/s00415-0050916-7

9.Driver JA, Kurth T, Buring JE, Gaziano JM, Logroscino G. Parkinson disease and risk of mortality: A prospective comorbidity-matched cohort study. Neurology 2008;70:1423-30. https://doi.org/10.1212/01.wnl.0000310414.85144.ee

10.Xu J, Gong DD, Man CF, Fan Y. Parkinson's disease and risk of mortality: Meta-analysis and systematic review. Acta Neurol Scan 2014;129:71-9. https://doi.org/10.1111/ane.12201

11.Pinter B, Diem-Zangerl A, Wenning GK, Scherfler C, Oberaigner W, Seppi K, et al. Mortality in Parkinson's disease: A 38-year follow-up study. Mov Dis 2015;30:266-9. https://doi.org/10.1002/mds.26060

12.Scorza FA, Cavalheiro EA, Scorza CA, Ferraz HB. Sudden unexpected death in Parkinson's disease: Perspectives on what we have learned about sudden unexpected death in epilepsy (SUDEP). Epilep Behav

https://doi.org/10.1016/i.yebeh.2016.01.035

$2016 ; 57: 124-5$

13. Matsumoto $H$, Sengoku R, Saito $Y$, Kakuta $Y$, Murayama S, Imafuku I. Sudden death in Parkinson's disease: A retrospective autopsy study. J Neurol Sci 2014;343:149-52.

https://doi.org/10.1016/j.jns.2014.05.060

14.Silva AS, Ariza D, Dias DPM, Crestani CC, Martins-Pinge MC. Cardiovascular and autonomic alterations in rats with Parkinsonism induced by 6-OHDA and treated with L-DOPA. Life Sci 2015;127:82-9. https://doi.org/10.1016/j.Ifs.2015.01.032

15.Scorza FA, Scorza CA, Ferraz Ii HB. Domperidone, Parkinson disease and sudden cardiac death: Mice and men show the way. Clinics 2016;71:59-61. https://doi.org/10.6061/clinics/2016(02)01

16.Renoux C, Dell'Aniello S, Khairy P, Marras C, Bugden S, Turin TC, et al. Ventricular tachyarrhythmia and sudden cardiac death with domperidone use in Parkinson's disease. Bri J Clin Pharmacol 2016;82:461-72. https://doi.org/10.1111/bcp.12964

17.Rajput AH, Rozdilsky B. Dysautonomia in Parkinsonism: A clinicopathological study. J Neurol Neurosur Psychiatr 1976;39:1092100. https://doi.org/10.1136/jnnp.39.11.1092

18. Alonso $A$, Huang $X$, Mosley $T H$, Heiss $G$, Chen $H$. Heart rate variability and the risk of Parkinson disease: The Atherosclerosis Risk in Communities study. Ann Neurol 2015;77:877-83. https://doi.org/10.1002/ana.24393 
19.Scorza FA, Fiorini AC, Scorza CA, Finsterer J. Cardiac abnormalities in Parkinson's disease and Parkinsonism. J Clin Neurosci 2018;53:1-5. https://doi.org/10.1016/j.jocn.2018.04.031

20.Sato K, Hatano T, Yamashiro K, Kagohashi M, Nishioka K, Izawa N, et al. Prognosis of Parkinson's disease: Time to stage III, IV, V and to motor fluctuations. Mov Dis 2006;21:1384-95.

https://doi.org/10.1002/mds.20993

21.Wang G, Li XJ, Hu YS, Cheng Q, Wang CF, Xiao Q, et al. Mortality from Parkinson's disease in China: Findings from a five-year follow up study in Shanghai. Can J Neurol Sci 2015;42:242-7. https://doi.org/10.1017/cjn.2015.49

22.Scorza FA, Cavalheiro EA, Scorza CA, Ferraz HB. Sudden unexpected death in Parkinson's disease: Perspectives on what we have learned about sudden unexpected death in epilepsy (SUDEP). Epilep Behav

https://doi.org/10.1016/j.yebeh.2016.01.035

$2016 ; 57: 124-5$

23. Nishida N, Yoshida K, Hata Y. Sudden unexpected death in early Parkinson's disease: neurogenic or cardiac death? Cardiovascul Pathol 2017;30:19-22. https://doi.org/10.1016/j.carpath.2017.06.001

24.Scorza FA, Tufik S, Scorza CA, Andersen ML, Cavalheiro EA. Sudden unexpected death in Parkinson's disease (SUDPAR): sleep apnea increases risk of heart attack. Sleep Breat 2017;21:965-6. https://doi.org/10.1007/s11325-017-1511-8

25.Scorza FA, Fiorini AC, Scorza CA, Finsterer J. Sudden unexpected death in Parkinson's disease (SUDPAR): A fatal event that James Parkinson did not address. Age Ageing 2018;47:627. https://doi.org/10.1093/ageing/afy064

26.Scorza FA, Fiorini AC, de Almeida ACG, Scorza CA, Finsterer J. In brief: Sudden unexpected death in Parkinson's disease. Acta Neurol Scan 2018;138:264-5. https://doi.org/10.1111/ane.12971

27. Rodrigues LD, Oliveira LF, Shinoda L, Scorza CA, Faber J, Ferraz HB, et al. Cardiovascular alterations in rats with Parkinsonism induced by 6-OHDA and treated with Domperidone. Sci Rep 2019;9. https://doi.org/10.1038/s41598-019-45518-z

28.Nejm MB, Andersen ML, Tufik S, Finsterer J, Scorza FA. Sudden death in Parkinson's disease: Unjustifiably forgotten. Parkinsonis Relat Dis 2019;58:88-9. https://doi.org/10.1016/j.parkreldis.2018.08.012 29.Zhang Y, Wang G. Response to: Sudden death in Parkinson's disease: Unjustifiably forgotten. Parkinsonis Relat Dis 2019;58:87. https://doi.org/10.1016/j.parkreldis.2018.08.013

30.Nejm MB, Andersen ML, Tufik S, Finsterer J, Scorza FA. Sudden unexpected death in Parkinson's disease: why do neuroscientists still ignore this condition? Neurol Sci 2019;40:413-4. https://doi.org/10.1007/s10072-018-3581-1

31.Menezes-Rodrigues FS, Scorza CS, Fiorini AC, Caricati-Neto A, Scorza CA, Finsterer J, et al. Sudden unexpected death in Parkinson's disease: why is drinking water important? Neurodegen Dis Manag 2019;9:241-6. https://doi.org/10.2217/nmt-2019-0010 
32. Rodrigues LD, Oliveira LF, Scorza CA, Andersen ML, Tufik S, Finsterer J, et al. REM sleep without atonia as prodromal marker of Lewy body disease: Fake news or the real deal? Parkinsonis Relat Dis 2019;67:34-5. https://doi.org/10.1016/j.parkreldis.2019.09.017

33. Rodrigues LD, Oliveira LF, Scorza CA, Andersen ML, Tufik S, Finsterer J, et al. We never speak about sudden unexpected death in Parkinson's disease. Eur J Neurol 2020;27:e30. https://doi.org/10.1111/ene.14217

34. Alves M, Caldeira D, Ferro JM, Ferreira JJ. Reply to the letter 'We never speak about sudden unexpected death in Parkinson's disease. Eur J Neurol 2020;27:e29. https://doi.org/10.1111/ene.14216

35.Scorza FA, de Almeida ACG, Scorza CA, Fiorini AC, Finsterer J. Proinflammatory Cytokines and Sudden Death in Parkinson's Disease: a Missing Piece of the Jigsaw Puzzle. J Neuroim Pharmacol 2020;15:5701. https://doi.org/10.1007/s11481-020-09950-7

36. Rodrigues LD, Oliveira LF, Scorza CA, Scorza FA, Andersen ML, Tufik $\mathrm{S}$, et al. Sudden unexpected death in Parkinson's disease: Who would think of the thyroid gland? Parkinsonis Relat Dis 2020;81:54-5. https://doi.org/10.1016/j.parkreldis.2020.10.010

37.Scorza FA, de Almeida ACG, Cysneiros RM, Scorza CA, Finsterer J. Letter to the editor: sudden death in Parkinson's disease: treating hypertension in the elderly is essential. Exp Opin Pharmacother 2021:1-2. https://doi.org/10.1080/14656566.2021.1833611

38.Jost WH. Reply to: Sudden Death In Parkinson's Disease: Treating Hypertension In The Elderly. Exp Opin Pharmacother 2020. https://doi.org/10.1080/14656566.2021.1833606

39.Micheli F, Gatto E, Fernandez Pardal M, Paradiso G. Domperidone and Parkinson's disease. Medicina 1988;48:218. https://doi.org/10.1007/978-3-642- 73899-9 20

40.Lertxundi U, Echaburu SD-, Soraluce A, Garcia M, Osante BR-, Aguirre C. Domperidone in Parkinson's Disease: A Perilous Arrhythmogenic or the Gold Standard? Curr Drug Saf 2013;8:63-8. https://doi.org/10.2174/1574886311308010009

41.Bacchi S, Chim I, Kramer P, Postuma RB. Domperidone for Hypotension in Parkinson's Disease: A Systematic Review. J Parkinsons Dis 2017;7:603-17. https://doi.org/10.3233/JPD-171209

42.Rossi M, Giorgi G. Domperidone and Long QT Syndrome. Curr Drug Saf 2010;5:257-62. https://doi.org/10.2174/157488610791698334 43. Heranval A, Lefaucheur R, Fetter D, Rouille A, le Goff F, Maltete D. Drugs with potential cardiac adverse effects: Retrospective study in a large cohort of parkinsonian patients. Rev Neurologiq 2016;172:31823. https://doi.org/10.1016/j.neurol.2015.11.007

44.Deguchi K, Sasaki I, Tsukaguchi M, Kamoda M, Touge T, Takeuchi $\mathrm{H}$, et al. Abnormalities of rate-corrected QT intervals in Parkinson's disease - A comparison with multiple system atrophy and progressive supranuclear palsy. J Neurol Sci 2002;199:31-7. https://doi.org/10.1016/S0022-510X(02)00079-5

45. Friedrich $C$, Rüdiger $H$, Schmidt $C$, Herting $B$, Prieur $S$, Junghanns $S$, et al. Baroreflex sensitivity and power spectral analysis in different 
extrapyramidal syndromes. J Neural Trans 2008;115:1527-36. https://doi.org/10.1007/s00702-008-0127-3

46.Finsterer J, Wahbi K. CNS-disease affecting the heart: Brain-heart disorders. J Neurol Sci 2014;345:8-14. https://doi.org/10.1016/j.jns.2014.07.003

47. Oka H, Mochio S, Sato H, Katayama K. Prolongation of QTc interval in patients with parkinson's disease. Eur Neurol 1997;37:186-9. https://doi.org/10.1159/000117432 